\title{
New planar trace humidity sensor
}

\author{
Carlo Tiebe ${ }^{1}$, Thomas Hübert ${ }^{1}$, Andreas Lorek ${ }^{2}$, Roland Wernecke ${ }^{3}$ \\ ${ }^{1}$ BAM Federal Institute for Materials Research and Testing, Unter den Eichen 87, 12205 Berlin, \\ Germany \\ corresponding author's e-mail: thomas.huebert@bam.de \\ ${ }^{2}$ German Aerospace Center (DLR), Rutherfordstraße 2, 12489 Berlin, Germany \\ ${ }^{3}$ dr. wernecke Feuchtemesstechnik GmbH, Gerlachstraße 41, 14480 Potsdam, Germany
}

\begin{abstract}
A new planar sensor element for continuous coulometric trace humidity measurements in industrial gases has been developed. In order to ensure precise measurements a calibration facility including a precision dew point hygrometer as a reference device was developed. The sensor can measure the humidity in the frost point temperature range of $-20^{\circ} \mathrm{C}$ to $-80^{\circ} \mathrm{C}$ and has an expanded uncertainty of $2 \mathrm{~K}$, a fast reaction time and a settling time of the entire system from 15 to $30 \mathrm{~min}$.
\end{abstract}

Key words: trace humidity, coulometric principle, HUMITRACE sensor, humidity measurement

\section{Introduction}

The trace humidity of gases covers frost point temperatures $t_{\mathrm{f}}$ below $-13{ }^{\circ} \mathrm{C}$ (equivalent to water vapour volume fraction $w_{v}$ smaller than $2000 \mathrm{ppm}_{\mathrm{v}}$ or absolute humidity $d_{\mathrm{v}}$ below $\left.1.45 \mathrm{~g} \cdot \mathrm{m}^{-3}\right)$. It is an important parameter of many gaseous precursors, final products and used carrier gases in chemical production, semiconductor industry and aerospace technology. There is a permanent need for online measurement of humidity as a quality parameter during the manufacturing process of these goods.

Coulometric sensor is a very robust and costeffective tool for determination of trace humidity. The sensor was first described by Keidel [1] and several commercial available advanced devices are based on this principle $[2,3]$. A traditional coulometric sensor is extensively prepared and consists of bifilar wires wound on a glass rod. The noble metal electrodes were coated with a thin homogeneous tetraphosphorus decaoxide $\left(\mathrm{P}_{4} \mathrm{O}_{10}\right)$ film as absorbents for water and as electrolyte. In the reaction with water, $\mathrm{P}_{4} \mathrm{O}_{10}$ is hydrolyzed and the formed phosphoric acid is electrolyzed at a voltage of at least $2 \mathrm{~V}(\mathrm{DC})$ on the electrodes to form hydrogen and oxygen. The measured current $(I)$ is proportional to the water content in the gas according to the FARADAY's law $[4,5]$ :

$Q=I \cdot \Delta t=F \cdot \frac{m_{v}}{M_{v}} \cdot z$ where $Q$ is the amount of charge in $A \mathrm{~s}, I$ the current in $A, \Delta t$ the time in $s, F$ the FARADAY constant $96485.3 \mathrm{~A} \cdot \mathrm{s} \cdot \mathrm{mol}^{-1}, m_{\mathrm{v}}$ the mass of converted water in $\mathrm{g}, M_{\mathrm{v}}$ the molar mass of water and $z$ the number of liberated electrons per converted water molecule.

However, in case of continuous measurements in a gas stream, the measured current depends on the gas flow rate and not always a complete electrolytic decomposition of all water molecules from the investigated gases can be realized, what may cause remarkable measurement uncertainties. Thus, for higher precision and traceability, the sensors themselves and the measurement procedures have to be improved and a calibration system is required. Therefore the aim of this development was to enhance coulometric trace humidity measurement by providing a cost effective sensor element which is insensitive against flow rate variations and which can be traceably calibrated.

\section{Experimental}

\subsection{HUMITRACE sensor}

The developed planar sensor (HUMITRACE sensor), emerged from the HUMITRACE technology transfer project [5], exhibited interdigital platinum electrodes each with 15 fingers of a widths of $200 \mu \mathrm{m}$ on an alumina substrate $(30 \times 10 \times 0.6 \mathrm{~mm})$ prepared by screen printing technology. The planar sensor element is protected by a porous polymer membrane to diminish the influence of a 
fluctuating gas flow rate. Water penetrates through the membrane and is absorbed by the hygroscopic coating on the noble metal electrodes followed by an electrolysis which generates a constant electrical current. Six exemplars of this sensor were investigated in five experimental runs in a humidity concentration range of four orders of magnitude.
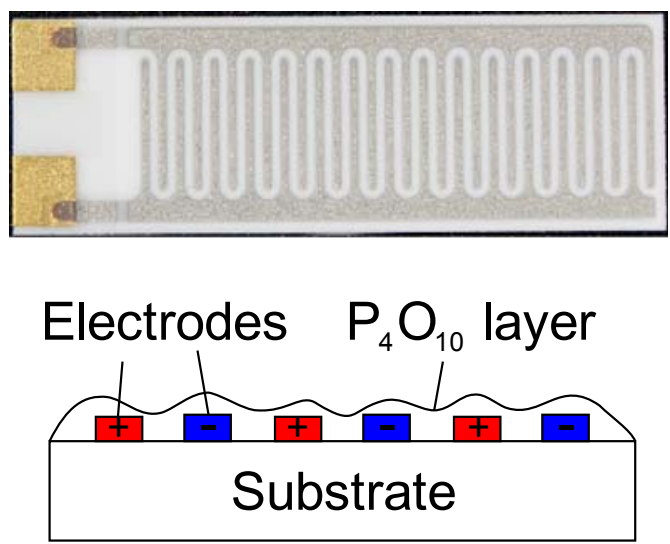

Figure 1: Plan view and scheme of the planar sensor element.

\subsection{Trace humidity calibration facility}

A test gas generation facility (THG11) was developed for sensor calibration, which provides a constant gas flow of defined trace humidity and permits to collect signals of six coulometric sensors simultaneously at atmospheric pressure. Compressed air is purified and dried by molecular sieve and absorption filters (Water Vapor Trap $5 \AA$, Sigma Aldrich and DAC-8, Boge Kompressoren). The atmospheric pressure is measured by the manometer LEO2 (Keller) with an uncertainty $u(p)$ of $0.1 \%$. Test gas is produced by mixing two gas streams - a dry and a water saturated gas stream of compressed air. The water saturation is realized by passing a gas flow through an impinger which is filled with pure water. The two gas streams were regulated by mass flow controllers (EL-FLOW ${ }^{\circledR}$, Bronkhorst). Whereby the standard uncertainty of each flow is $u(\mathrm{MFC})=0.5 \%$. The total test gas flow is split into seven test gas streams, six going above coulometric sensors and one flows into a precision dew point hygrometer (chilled mirror principle, S4000TRS, Michell Instruments) which is calibrated traceable to NIST standard and used as reference device. The hygrometer has frost point measurement range of $-80{ }^{\circ} \mathrm{C}$ to $-30{ }^{\circ} \mathrm{C}$ with an uncertainty $(\mathrm{k}=2)$ from $0.4 \mathrm{~K}$ to $0.6 \mathrm{~K}$ depending on the magnitude of the frost point temperature. The current is measured by a digital multimeter Agilent HP3458A $(u(I)=0.0014 \%)$. The gas flow control and the data acquisition are performed by an adapted software program (LabVIEW 2010, National Instruments).

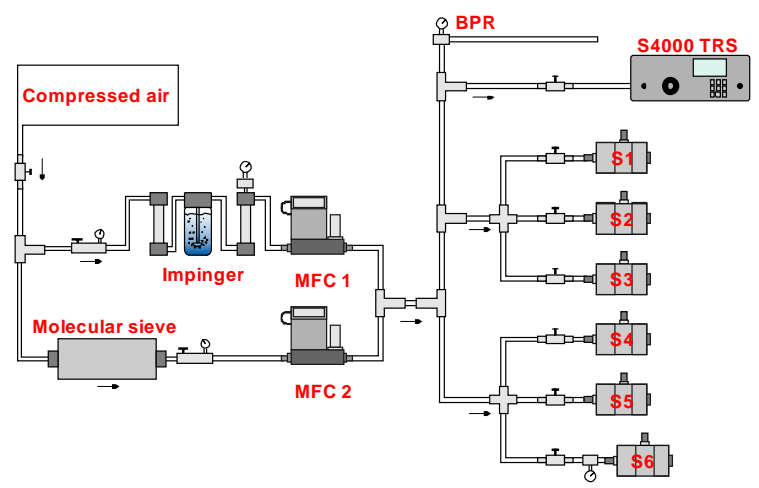

Figure 2: Scheme of the calibration facility (THG11), S1...S6 - Sensor 1 ...Sensor 6, BPR - back pressure regulator.

\subsection{Data Analysis}

According to FARADAY's law a linear relationship between water vapour concentration and electrical current is expected. However, due to many customers' requests, the results of trace humidity measurements shall be given in frost point temperatures, the temperature at which the prevailing water vapour partial pressure is equal to the saturation vapour pressure and at which ice begins to form. Furthermore, the primary measurement of the reference dew point hygrometer is also the frost point temperature. Therefore a calibration of the sensor current signal $(I)$ to frost point temperature $\left(t_{\mathrm{f}}\right)$ was performed assuming the following empiric nonlinear function:

$t_{\mathrm{f}}=b \cdot \ln (I)+a$

Based on equation (2), the uncertainty of the frost point temperature measured by coulometric sensor and the limit of detection was estimated [6]. The signal scattering of different HUMITRACE sensor samples was tested in a one-way analysis of variances (ANOVA) using Origin 8.1 (Origin Lab Corporation). The parameters $a$ and $b$ of six sensor calibration curves were analysed in an F-test for a confidence level of $95 \%[6,7]$.

The different measures for humidity were converted into each other, e. g. frost point temperature $t_{\mathrm{f}}$ into volume fraction $w_{\mathrm{v}}$ in $\mathrm{ppm}_{\mathrm{v}}$, by HumiCalc ${ }^{\circledR}$ (Thunder Scientific) based on SONNTAG equation [8]. 


\section{Results}

\subsection{Sensor response}

The signals of the six HUMITRACE sensors were evaluated five times in the range of frost point temperatures from $-80{ }^{\circ} \mathrm{C}$ to $-30{ }^{\circ} \mathrm{C}$ using the described laboratory facility THG11. As shown in Figure 3, the sensor current correlates with the humidity content of the air.

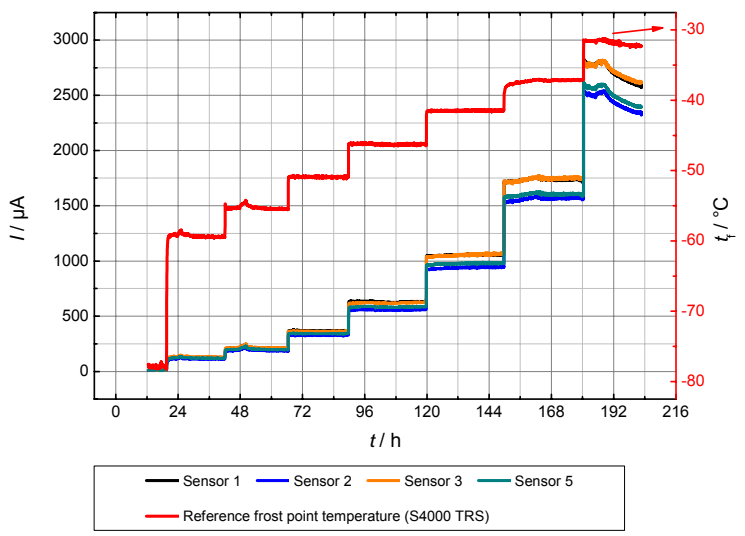

Figure 3: Signal curve of four coulometric sensors (left y-axis) and the reference humidity measurement (right $y$-axis) over measuring time, first run.

All these results were achieved without that the total amount of electric charge $(Q=I \cdot \Delta t)$ of 3600 Coulomb was exceeded. The results are comparable and reproducible up to this amount of charge. When this value is exceeded sensor regeneration is advisable. The HUMITRACE sensors are regenerated by cleaning the substrate surface and new preparation of the hygroscopic $\mathrm{P}_{4} \mathrm{O}_{10}$-layer.

\subsection{Calibration function}

The frost point temperature $\left(t_{\mathrm{f}}\right)$ in dependence on the current $(I)$ of the coulometric sensors is plotted in Figure 4.

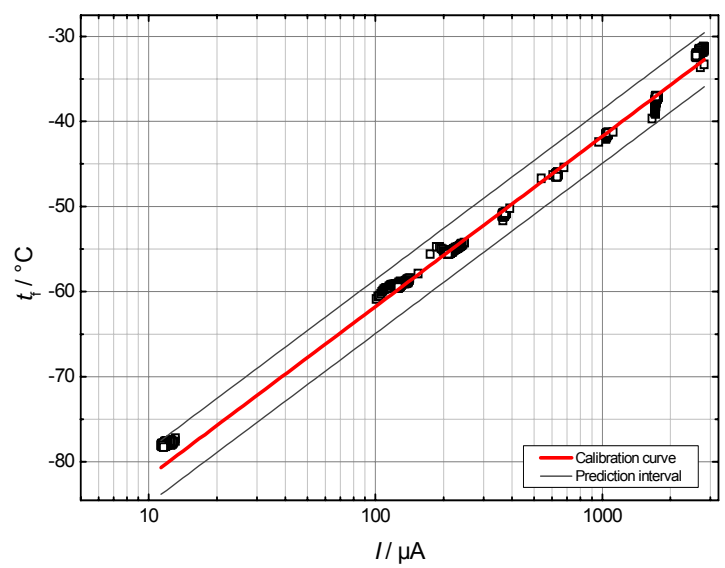

Figure 4: Calibration function of a coulometric trace humidity sensor (sensor 1, first run) for (20 \pm 2$) \mathrm{NL} / \mathrm{h}$ carrier gas flow of purified compressed air.
A calibration function can be calculated by a linear regression according to equation (2). The parameters $a$ and $b$ of the calibration functions of all six sensors were fitted and the results are listed in table 1. Different values were obtained indicating an apparent individual behaviour of each sensor.

Table 1: Nonlinear calibration function parameters of the coulometric sensors $\left(t_{f}=b \cdot \ln (I)+a\right)$.

\begin{tabular}{ccccccc}
\hline \multicolumn{7}{c}{ Parameter a } \\
No. & S1 & S2 & S3 & S4 $^{*}$ & S5 & S6 \\
\hline 1 & $-100,227$ & $-98,787$ & $-101,355$ & $(-114.445)$ & $-100,682$ & $-101,310$ \\
2 & $-102,470$ & $-101,053$ & $-103,771$ & $(-114.144)$ & $-103,259$ & $-104,349$ \\
3 & $-101,012$ & $-100,370$ & $-101,369$ & $(-105.377)$ & $-101,583$ & $-100,762$ \\
4 & $-102,884$ & n. d. & $-103,909$ & $(-113.961)$ & $-101,353$ & $-103,707$ \\
5 & $-102,812$ & n. d. & $-103,159(-106.284)$ & $-103,029$ & $-105,226$ \\
\hline
\end{tabular}

\begin{tabular}{ccccrcc}
\hline & \multicolumn{5}{c}{ Parameter $\boldsymbol{b}$} \\
No. & S1 & S2 & S3 & \multicolumn{1}{c}{ S4 $^{\boldsymbol{*}}$} & S5 & S6 \\
\hline 1 & 8,468 & 8,398 & 8,633 & $(-10.681)$ & 8,630 & 8,686 \\
2 & 8,841 & 8,763 & 8,990 & $(-9.783)$ & 9,069 & 9,092 \\
3 & 8,532 & 8,779 & 8,566 & $(-7.964)$ & 8,702 & 8,331 \\
4 & 8,893 & n. d. & 9,073 & $(-9.354)$ & 8,704 & 9,010 \\
5 & 8,814 & n. d. & 8,923 & $(-8.257)$ & 8,900 & 9,183 \\
\hline
\end{tabular}

n. d. - not determined

S1...S6 - Sensor 1 ... Sensor 6

* - sensor without polymer membrane

\subsection{Uncertainty of Measurements}

A linear regression based of all data from the six sensors in five runs according to equation (2) results in an expanded uncertainty of the frost point temperature of $2 \mathrm{~K}$. This implies that all investigated sensors do not differ from each other. Therefore the variances of the parameters of five sensors with polymer membrane given in table 1 were analysed to verify this assumption. The critical $F$-value of the ANOVA is 2.809 at the confidence level of $95 \%$. The obtained $F$-value of parameter $a$ is 1.876 and of parameter $b$ is 0.410 . Both parameters are smaller than the critical $F$-value. This statistical evidence coincides with the null hypothesis that the sensor parameters $a$ and $b$ of the calibration curve for the sensors are not different from each other at $95 \%$ confidence level. Therefore it can be concluded that the sensors exhibit no unique response behaviour and one calibration function can be used for all sensors.

Further, the frost point temperature detection limit of these measurements was estimated to be $(-80.6 \pm 2.7){ }^{\circ} \mathrm{C}$ corresponding to a current of $(12.1 \pm 3.9) \mu \mathrm{A}$.

\subsection{Dynamic behaviour}

Figure 5 illustrates the dynamic behaviour of the entire system - THG11, coulometric sensors and precision dew point hygrometer. For a decreasing humidity change of frost point temperatures from $-31.2{ }^{\circ} \mathrm{C}$ to $-78.8{ }^{\circ} \mathrm{C}$ (equal to $332 \mathrm{ppm}_{\mathrm{v}}$ to $\left.0.66 \mathrm{ppm}_{\mathrm{v}}\right)$ a recovery time $\left(t_{10}\right)$, 
the time when a sensor gains $10 \%$ of its initial value was 5 to $10 \mathrm{~min}$.

An increase of humidity from $-78.8{ }^{\circ} \mathrm{C}$ to $-30.8^{\circ} \mathrm{C}$ frost point temperature (equal to $0,66 \mathrm{ppm}_{\mathrm{v}}$ to $342 \mathrm{ppm}_{\mathrm{v}}$ ) results in response times $\left(t_{90}\right)$ of 10 to $15 \mathrm{~min}$.

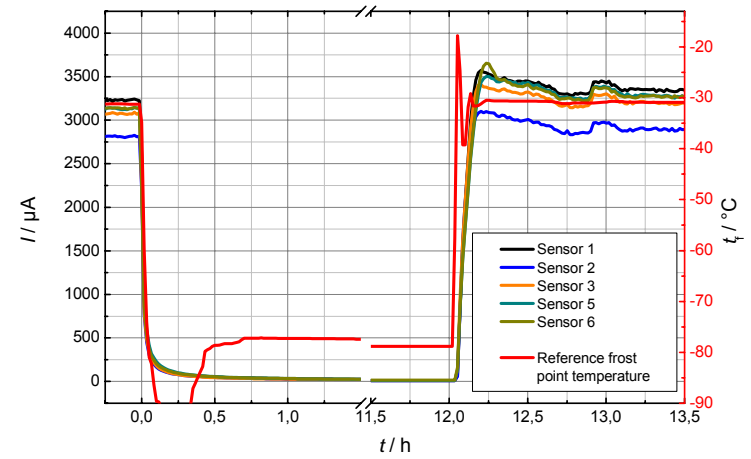

Figure 5: Coulometric sensor for two humidity changes, first from $t_{f}=-31.2{ }^{\circ} \mathrm{C}$ to $-78.8^{\circ} \mathrm{C}$ and then from $-78.8^{\circ} \mathrm{C}$ to $-30.4{ }^{\circ} \mathrm{C}$.

In general, the entire system exhibits an average response time for rising humidity in the range of 15 to $30 \mathrm{~min}$ and recovery time at decreasing humidity is 10 to $30 \mathrm{~min}$.

\section{Conclusions}

The new HUMITRACE sensors can be manufactured in a cost effective way and grant in regard to the calibration facilities a higher precision and reliability of trace humidity measurements.

From the one-way ANOVA, the parameters of the calibration functions are not significantly different and one calibration curve can be used for all sensor samples. The sensor element can be continuously used for humidity measurements in compressed air up to 3600 Coulomb or up to 12 month, respectively without regeneration.

The dynamic behaviour of the entire system (test gas generation facility (THG11), the coulometric sensors and the precision dew point hygrometer) can be characterized by a response time $\left(t_{90}\right)$ in the range of 15 to $30 \mathrm{~min}$ and a recovery time $\left(t_{10}\right)$ of 10 to $30 \mathrm{~min}$.

\section{Acknowledgements}

The authors thank Heidi Lorenz, Ulrich Banach and Volker Schwanke for technical support as well as the Federal Ministry of Economics and Technology of Germany (BMWi) for financial support - MNPQ-project No. 20/09.

\section{References}

[1] F. A. Keidel, Determination of Water by Direct Amperometric Measurement, Analytical Chemistry 13, $2043-2048$ (1959).

[2] T. Hübert, W. Schuffenhauer, R. Wernecke, Selfmonitoring and regenerating system for on-line trace moisture measurements in chemical process gases, Chemie Ingenieur Technik 72, 1380-1382 (2000).

[3] J. R. Riddle, Spurenfeuchte von Gasen zuverlässig messen, GIT Labor-Fachzeitschrift 4, 242-243 (2011)

[4] T. Hübert, A. Lorek, R. Wernecke, Quality Assurance for Industrial Coulometric Trace Humidity Measurement, Proc. of the $4^{\text {th }}$ Intern.Symp. on Humidity and Moisture 16-19 September 2002, Taipei, 157.

[5] A. Lorek, A. Koncz, R. Wernecke, Development of a gas flow independent coulometric trace humidity sensor for aerospace and industry Proc. of Aquametry 2010 in Weimar, 289-296.

[6] K. Danzer, Analytical Chemistry - Theoretical and Metrological Fundamentals, Springer-Verlag Berlin-Heidelberg (2007).

[7] NIST/SEMATECH e-Handbook of Statistical Methods, http://www.itl.nist.gov/div898/handbook/ (2006-01-05).

[8] D. Sonntag, Advancements in the field of hygrometry, Meteorol. Zeitschrift N. F. 3, 51-66 (1994). 